\title{
A Vulnerable Scoring through Code-based Cloud Storage System with Sheltered Data Forwarding
}

\author{
S.Palani \\ PG Scholar \\ Department of CSE \\ SKP Engineering College \\ Tiruvannamalai.
}

\author{
A.Kumaresan \\ Professor, \\ Department of CSE \\ SKP Engineering College \\ Tiruvannamalai.
}

\author{
S.Murali \\ Assistant Professor \\ School of Computing \\ Science and Engineering, \\ VIT University, Vellore.
}

\begin{abstract}
Cloud Storage System has a collection of storage servers provides long-standing storage services over the internet. Data privacy becomes a major concern in cloud storage system because user stores their data in third party cloud system. An encryption scheme available for data privacy but it limits the number of functions done in storage system. Building a secure storage system that supports multiple functions is tough when the storage system is distributed and has no central authority. A new idea is proposed proxy re-encryption scheme for decentralizes erasure code for defending the distributed system. The distributed storage system not only supports secure and robust data storage and recovery, but client onward his information in the storage servers to another user without retrieving the data back. The important technical part is that the proxy re-encryption scheme supports encoding operations over encrypted data as well as forwarding operations over encoded and encrypted data. Our schemes fully integrate encoding, encrypting, and onward.
\end{abstract}

\section{General Terms}

Algorithms, Security, Cloud storage, and Distributed systems

\section{Keywords}

Ciphertext, Plaintext Key translation, Proxy re-encryption.

\section{INTRODUCTION}

As very fast networks and ever present internet entry become obtainable in nowadays, more services are providing in the internets clients or users can use at anytime from anywhere. Cloud computing is a long standing storage server and also providing the services to the users. A cloud storage data in other storage server's cloud system causes grim anxiety on the data confidentiality highly secure to provide the messages in the storages severs. The users are using the cryptographic method for encrypt messages and store the messages. The client need the messages, clients get the key from storage sever and use cryptographic keys for decryption.

Here, the client lost the keys for the particular data, the server side will rise the some security issues and the client will give the exact solution to sever. Once again the key provide to the client. In previous process are the bulk of data transfer between the client and server. Proxy re-encryption allows a proxy to transform a cipher text computed under Alice's public key into one that can be opened by Bob's secret key. There are many useful applications of this primitive. For instance, Alice might wish to temporarily forward encrypted email to her colleague Bob, without giving him her secret key. In this case, Alice the delegator could designate a proxy toreencrypt her incoming mail into a format that Bob the delegate can decrypt using his own secret key. Alice could simply provide her secret key to the proxy, but this requires an unrealistic level of trust in the proxy [1]. It has more drawbacks. Those are given below:

- The victory in hacking of data transfer from other storage server.

- Lot of time loss for data transmission.

- More communication traffic occurs.

The integrated monitoring has become an important approach for investigation, detection, and policy decision in many fields. Unfortunately, current monitoring systems are commonly developed by different organizations using specific technologies and platforms, bringing a lot of difficulties for the seamless integration and unified access. Through the integration architecture, the distributed isomerous monitoring systems can be accessed in a uni-fied user interface if owning the corresponding ranking. In order to validate the proposed architecture, three different monitoring systems are constructed and integrated [2].

The main technical contribution is that the proxy reencryption scheme supports encoding operations over encrypted messages as well as forwarding operations over encoded and encrypted messages. This method fully integrates encrypting, encoding, and forwarding [3]. OceanStore is a utility infrastructure designed to span the globe and provide continuous access to persistent in formation. Since this infrastructure is comprised of un-trusted servers, data is protected through redundancy and cryptographic techniques. To improve performance, data is allowed to be cached anywhere, anytime. Additionally, monitoring of usage patterns allows adaptation to regional outages and denial of service attacks; Drawback of this paper is proper prototype model not developed [4].

PAST is a peer-to-peer Internet application and is entirely self-organizing. PAST nodes serve as access points for clients, participate in the routing of client requests, and contribute storage to the system. Nodes are not trusted, they may join the system at anytime and may silently leave the system without warning [5]. While PAST offers persistent storage services, its access semantics differ from that of a conventional file system. Files stored in PAST are associated with a quasi- 
unique fileId that is generated at the time of the file's insertion into PAST. In large part, Farsite's design is a careful synthesis of techniques that are well known within the systems and security communities, including replication, Byzantine-faulttolerance, cryptography, certificates, leases, client caching, and secret sharing. However, we have also developed several new techniques to address issues that have arisen in Farsite's design: Convergent encryption permits identifying and coalescing duplicate files encrypted with different users' keys. Exclusive encryption enforces filename syntax while maintaining filename privacy. Scalable, distributed, faulttolerant database supports distributed duplicate-file detection. We use a novel combination of secret sharing, message authentication codes, and logging to enable secure crash recovery [6]. Glacier's approach is 'extreme' in the sense that it does not rely on introspection, which has inherent limitations in its ability to capture all sources of correlated failures; instead, it uses massive redundancy to mask the effects of large-scale correlated failures such as worm attacks. The system uses erasure codes and garbage collection to mitigate the storage cost of redundancy and relies on aggregation and a loosely coupled fragment maintenance protocol to reduce the message costs [7].

\section{SYSTEM ARCHITECTURE}

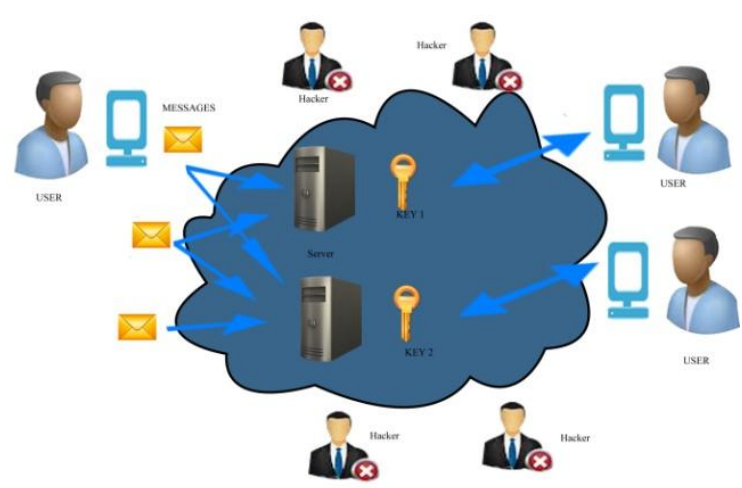

Figure 2.1 System Architecture Diagram

The client sends the request to the server. The server receives the request and creates a key for particular data. And the server only send key to the client, the key is very highly secure. Client gets the key and fetches the data from the cloud with the help of key.

\subsection{Construction of Cloud Data Storage}

The Admin process the admin can login to give his username and password. Then the server system process can be opened. In server system process the admin first see the remote servers Ip-address for send that Ip-address to the receiver.

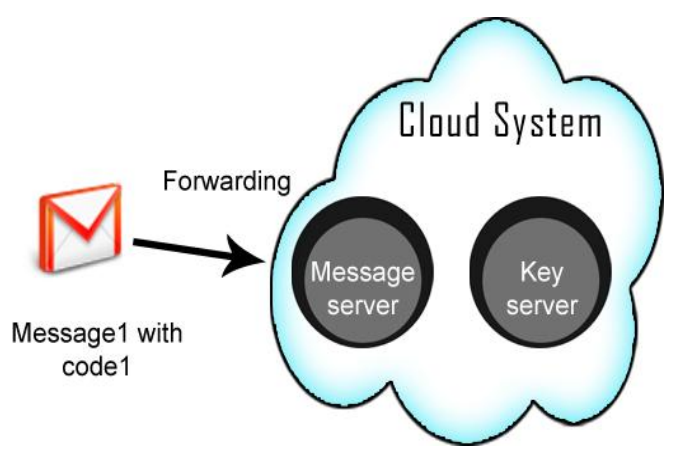

Figure 2.1.1 Build a Cloud System
Then the server can omit the process to activate or de-activate the execution. For activating the process the storage server can exhibit the Ip-address. For de-activating the process the storage server cannot display the Ip-address. The activated Ipaddresses are stored in obtainable storage server.

\subsection{Data Encryption Portion}

Cloud login process the user can login own information. If the user don't have the account for that cloud system first the user can register information for using and toward the inside the cloud system. After entering the registration process the information can be stored in database of the cloud system. In file upload process the user has to choose one file from browsing the system. Now, the server from the cloud can give the encrypted form of the uploading file.

\subsection{Data Onward Process}

The Onward process first we can see the storage information for the uploaded files. If the onward column display the onward value is true the user cannot onward to another person. If the onward column display the forwarded value is false the user can forward the file into another person. Then the current user has login to the cloud system and to verify the received details.

\subsection{Retrieval Process}

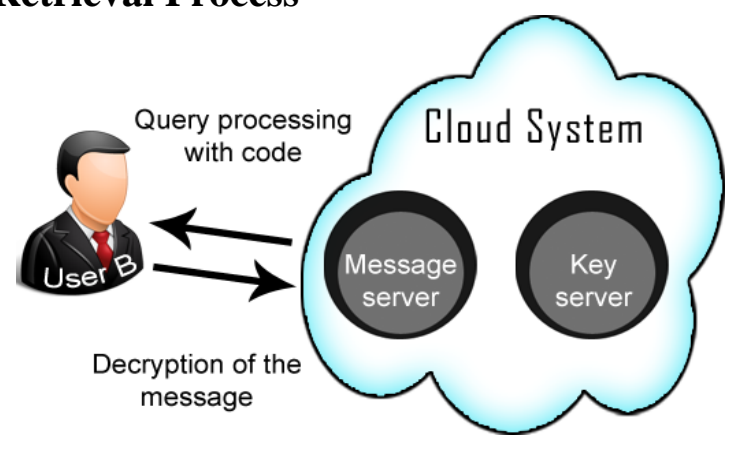

Figure 2.4.1 Receiving the data from Cloud

First, the server process can be run which means the server can be linked with its particular user. Now, the user has to download the file to download the file key. In file key downloading process have several code words. Then using that key the client can view the file and use that file appropriately.

\section{Results and discussions}

In this section, we discuss potential attacks against either the durability or the integrity of data stored in server. Since the cloud storage system is providing the security to the data. But the success hackers get the data between client and server data transfer. Here implementing the sheltered data forwarding and only send the key. So the data is highly confidential and secure. 


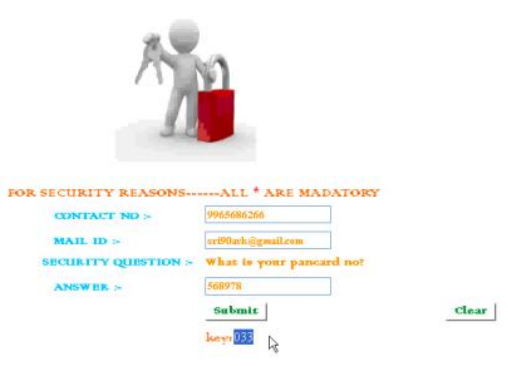

Figure 3.1 Build a Secure key

Using a secret encryption key that is stored in user database, sever can encrypt a document that you are posting in a private database, provided the document contains fields that are encryptable.

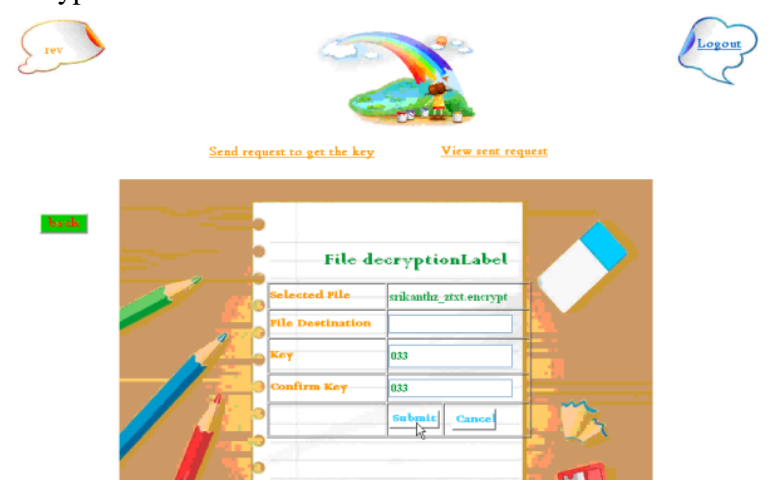

Figure 3.2 key conformation

Each data have a key in the database Server check the conform key and submit to the particular client.

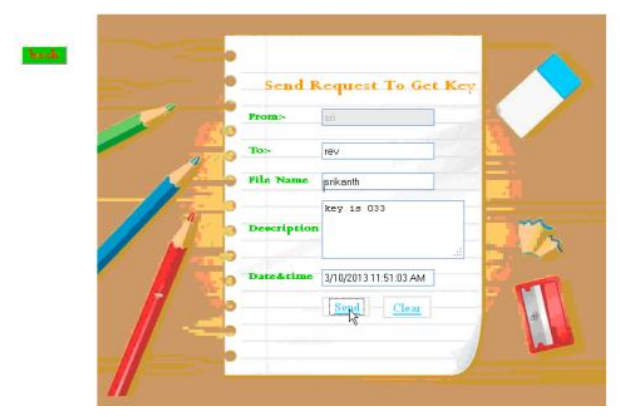

Figure 3.3 Transfer a key

Only the sender and intended receiver should be able to understand keys. The receiver must submit key and get the data from the database.

\section{CONCLUSION}

The focus of this paper is to provide a well-defined data retrieving and storage between the user and cloud server. We use a combination of secret sharing, logging to enable secure crash recovery and message authentication codes. It there is little consideration on the security and privacy such as data encryption, decryption, and so on. The secure data exchanging and system integration will be concentrated in the future.

\section{ACKNOWLEDGEMENTS}

We thank Mr. K. Vijayan, Mr. S. Muneer Ahamed, Mr. E.Pushparaj and Mr. P.Rajesh for helpful discussions and comments.

\section{REFERENCES}

[1] Giuseppe Ateniese, Kevin fu, Amherst Matthew Green, Susan Hohenberger, February 2006. Improved Proxy Re-encryption Schemes with Applications to Secure Distributed Storage. The Johns Hopkins University, University of Massachusetts, The Johns Hopkins University and Massachusetts Institute of Technology.

[2] Zhongwen Guo, Member, IEEE, Pengpeng Chen, Hao Zhang, , Chunrong Li and Mingxing Jiang. May 2012. IMA: An Integrated Monitoring Architecture With Sensor Networks. A Secure Erasure Code-Based Cloud Storage System with Secure Data Forwarding,

[3] J. Kubiatowicz, D. Bindel, Y. Chen, P. Eaton, D. Geels, R. Gummadi, S. Rhea, H. eatherspoon, W. Weimer, C. Wells, and B. Zhao, 2000. "Oceanstore: An Architecture for Global-Scale Persistent Storage," Proc. Ninth Int'l Conf. Architectural Support for Programming Languages and Operating Systems (ASPLOS), pp. 190201.

[4] P. Druschel and A. Rowstron, 2001. "PAST: A LargeScale, Persistent Peer-to-Peer Storage Utility," Proc. Eighth Workshop Hot Topics in Operating System (HotOS VIII), pp. 75-80.

[5] A. Adya, W.J. Bolosky, M. Castro, G. Cermak, R. Chaiken, J.R. Douceur, J. Howell, J.R. Lorch, M. Theimer, and R. Wattenhofer, "Farsite: Federated, 2002. Available, and Reliable Storage for an Incompletely Trusted Environment," Proc. Fifth Symp. Operating System Design and Implementation (OSDI), pp. 1-14.

[6] A. Haeberlen, A. Mislove, and P. Druschel, 2005. "Glacier: Highly Durable, Decentralized Storage Despite Massive Correlated Failures," Proc. Second Symp. Networked Systems Design and Implementation (NSDI), pp. 143-158. 